\title{
Konsequent privat - Folgen der Gesundheitspolitik für das deutsche Krankenhauswesen Ohne wirtschaftliches Denken geht das Gesundheitssystem bankrott
}

In Deutschland ist man stolz auf das ausgesprochen hohe Maß an Solidarität unseres Gesundheitswesens. Die Finanzierung mit einkommensabhängigen Beiträgen bei beitragsunabhängigen Leistungen impliziert jedoch auch Fehlanreize. Viele Jahre lang hat sich dieses System dennoch getragen. Die Bevölkerungsstruktur mit vielen sozialversicherungspflichtigen Arbeitnehmern bei verhältnismäßig wenigen Leistungsempfängern hielt das System der Umlagefinanzierung am Laufen. Nun müssen wir uns einer neuen Herausforderungen stellen: der Finanzierung eines rasanten medizinisch-technischen Fortschritts bei gleichzeitiger Alterung der Bevölkerung.

Das Gesundheitswesen ist einer der dynamischsten Wachstumsmärkte in Deutschland. Dies erfordert von den medizinischen Leistungserbringern ein besonders innovatives Management. Es kann aber nur dann seine vollen Früchte tragen, wenn die Politik die entsprechenden Voraussetzungen schafft. Soll der Gesundheitsbereich weiterhin ein Wachstumsmotor bleiben, sind nachhaltige und zukunftsorientierte Finanzierungsmodelle notwendig, die dies erlauben. Die letzte Gesundheitsreform jedoch ebnete den Weg in ein zentralistisches Gesundheitswesen. Die gesetzlichen Vorgaben liefern weder eine Lösung der Einnahmeproblematik der gesetzlichen Krankenversicherung noch belohnen sie effizientes Arbeiten, was wiederum die Investitionskraft schwächt.

\section{Rationalisierungsdruck für die Krankenhäuser}

Die Gesundheitsreform schlägt sich für die Krankenhäuser am deutlichsten in der Sanierungsabgabe nieder. Knapp 400 Millionen Euro tragen die Krankenhäuser zur finanziellen Unterstützung der gesetzlichen Krankenversicherung bei. Dies ist zwar weniger als der ursprünglich vorgesehene Betrag von 500 Millionen Euro, das Resultat aber bleibt das Gleiche: In Zusammenhang mit den weiteren Belastungen, die sich aus den neuen Tarifverträgen, dem Arbeitszeitrecht, der Mehrwertsteuererhöhung und der Energiekostenentwicklung er- geben, werden die Krankenhäuser in naher Zukunft unter einem erhöhten Kosten- und damit Rationalisierungsdruck stehen.

Trotz dieser Erkenntnis, die den verstärkten Einsatz betriebswirtschaftlicher Instrumente zur Folge haben müsste, zeichnen sich als Resultat der Gesundheitsreform Verstaatlichungstendenzen ab. Die in den 1990er-Jahren noch präferierte Selbstverwaltung wird zunehmend ausgehöhlt. Exemplarisch zeigt sich das bei der neuen Organisation des gemeinsamen Bundesausschusses. Schwerwiegender jedoch sind die Versäumnisse bei der Umsetzung eines neuen Finanzierungsmodells.

Die beschlossene Einführung des sogenannten Gesundheitsfonds ab 2009 wird zu einer Zentralisierung in der Krankenversicherung führen, an deren Ende die staatliche Einheitskasse stehen kann. Dieser Weg wird auch nicht ohne Auswirkungen auf die Selbstverwaltung im deutschen Gesundheitswesen bleiben, was sich dann auch auf die weitere Ausgestaltung des Fallpauschalensystems auswirken wird. Diese Änderungen setzen jedoch an der falschen Stelle an. Ein in seinen Grundstrukturen akzeptiertes und dem Grundkonsens der Solidarität verpflichtetes Finanzierungssystem des deutschen Gesundheitswesens wird damit seinem Ende nähergebracht, ohne die tatsächlichen Ursachen der Krise zu beheben.
Von anderen Punkten der Gesundheitsreform können die Krankenhäuser profitieren. Die Vereinfachung ihrer Zulassung zur ambulanten Leistungserbringung, die neuen Möglichkeiten des Vertragsärzteänderungsgesetzes und die pflegesatzrechtlichen Veränderungen ergeben auch Vorteile. Dennoch wirft das unbefriedigende Finanzierungsmodell seine Schatten auch auf diese positiv hervorzuhebenden Änderungen.

\section{Wettbewerb im Krankenhaus}

Für die weitere Entwicklung der Krankenhäuser wird es von entscheidender Bedeutung sein, inwieweit der Wettbewerbsgedanke im Gesundheitswesen tatsächlich umgesetzt und die Branche von bürokratischen Hürden befreit wird. Die Orientierung an privatwirtschaftlichen Grundprinzipien, die mit einer Deregulierung einhergehen muss, darf nicht mehr lange aufgeschoben werden. Schon jetzt ist der Druck auf viele Krankenhäuser enorm, ihre Existenz nicht bedingungslos garantiert.

Aus Sicht der Krankenhäuser zählt zu den wichtigsten Forderungen für eine neue Reform die zukünftige Ausgestaltung der Fallpauschalen, die als Festpreise definiert werden und in flexible, krankenhausindividuell verhandelbare Budgets eingebunden werden müssen. Dies setzt aber Dezentralisierung und lokale Handlungsspielräume voraus das Gegenteil der gegenwärtigen Politik.

\section{Die Rolle der privaten Kranken- versicherungen}

Die privaten Krankenversicherungen (PKV) sind eine wesentliche Stütze des deutschen Gesundheitswesens. Sie zählen zu den wichtigsten Kostenträgern und sind der Garant für eine pluralistische Struktur, die wiederum Voraussetzung für einen funktionierenden Wettbewerb ist. Dem Handeln der privaten Krankenversicherungen im Geschäftsalltag liegen betriebswirt- 
schaftliche Prinzipien zugrunde, die notwendig sind, um die Knappheit finanzieller Mittel möglichst optimal zu managen. Damit zeigen die PKV-Unternehmen auch, dass Solidarität nicht ausschließlich durch ein staatlich reguliertes Umverteilungssystem erreicht werden kann.

Im Gegenteil: Die PKV und ihre Versicherten tragen zur Finanzierung des gesamten Gesundheitswesens in Deutschland einen überproportionalen Teil bei. Sie sind damit ein wichtiger Motor für das kostenintensive Krankenhauswesen. Ihre Struktur und ihr Aufbau liefern darüber hinaus auch einen wichtigen Beitrag zur Sicherstellung der Investitionsfähigkeit der Krankenhäuser in Deutschland und damit für die Innovationskraft der gesamten Gesundheitsbranche. Im Ergebnis profitieren alle, auch die Patienten der gesetzlichen Krankenversicherung.

Die PKV-Versicherten spüren direkt, wie die Anwendung privatwirtschaftlicher Prinzipien die Gewährleistung von Patienteninteressen sichert: Leistungsgarantie, freie Arztwahl und unbehinderte Krankenhauswahl sowie Zugang zur Spitzenmedizin sind nur einige Beispiele, die vor dem Hintergrund der schleichenden Rationierung in der GKV verstärkt an Bedeutung gewinnen und durch die PKV in besonderem Maße gewährleistet werden.

\section{Stärkung des Prinzips von Leistung und Gegenleistung}

Das Festhalten am bisherigen Finanzierungssystem ist auf Dauer undenkbar. Eine wachsende Mehrheit der Bevölkerung kann nicht dauerhaft von einer schrumpfenden Minderheit getragen werden. Je länger am derzeitigen System der Umverteilung festgehalten wird, desto schmerzhafter werden die zwangsläufig auf uns zukommenden Veränderungen. Es wird nicht möglich sein, das generationenübergreifende Bewusstsein der Solidarität auch nachfolgenden Generationen zu vermitteln, wenn die Realität nicht anerkannt und die Regeln des Systems entsprechend angepasst werden.

Derzeit erhöht sich der Druck innerhalb des Systems. Die notwendigen Anpassungen der finanziellen Rahmenbedingungen unseres Gesundheitswesens erfolgen in immer kürzeren Abständen durch Gesundheitsreformen, die in kei- ner Weise nachhaltig sind. Statt den erfolgreichen Grundsätzen der privaten Krankenversicherung, also dem Prinzip von Leistung und Gegenleistung, zu folgen, wird am derzeitigen System festgehalten, dessen Fundamente werden gestärkt und damit die Ursachen der finanziellen Misere zementiert.

\section{Sichere Rahmenbedingungen für die Krankenversorgung}

Für das Krankenhauswesen wird das nicht ohne Folgen bleiben. Es stellt sich die unternehmerische Frage, wie künftig Investitionen platziert werden sollen, deren Return on Investment 10, 15 oder gar 20 Jahre benötigen wird, zugleich der gesetzliche Rahmen jedoch ständigen und nicht kalkulierbaren Veränderungen unterliegt.

Das Risiko von Fehlinvestitionen und weiteren Finanzmittel verzehrenden Verlusten kann nur gemindert werden, wenn klar ist, in welche Richtung sich das System wendet. Die Rahmenbedingungen müssen den Unternehmern im Gesundheitswesen zunächst Sicherheit vermitteln und letztlich auch Erfolg in Aussicht stellen. Derzeit ist das Ausmaß der Unsicherheit ein nicht unwesentlicher Einflussfaktor unternehmerischer Entscheidungen im Krankenhauswesen.

\section{Weniger ideologische Bremsen sind notwendig}

Der Wettbewerb unter den Krankenhäusern wird intensiver werden. Seriöse Studien gehen davon aus, dass mittelfristig bis zu einem Drittel der Krankenhausbetten aus der Krankenhausplanung herausfallen werden. Dieses neue Wettbewerbsumfeld mit all seinen Konsequenzen wird in der Bevölkerung nur dann positiv aufgenommen, wenn die Qualität glaubhaft gewährleistet und die Versorgungssicherheit nicht gefährdet ist. Dafür sind die notwendigen Vorkehrungen zu treffen, für die die Krankenhausbetreiber kräftig investieren müssen. Die Bereitschaft dazu ist gegeben. Es bedarf dafür jedoch einer Vertrauen schaffenden und Erfolg versprechenden Basis.

Die Aufgabe des Gesetzgebers ist es daher, für Rahmenbedingungen zu sorgen, die die notwendigen Spielräume für das Krankenhausmanagement mit dem Wunsch nach einer flächendeckenden und leistungsstarken Medizin verbin- den können. Die Krankenhäuser werden dann ihre Kompetenz in betriebswirtschaftlicher und medizinischer Hinsicht noch wesentlich stärker einbringen als bislang.

Inwieweit das auch in der Zukunft gelingt, hängt vom Mut der politischen Entscheidungsträger ab, dem Wettbewerb Vorrang vor einer Staatsmedizin einzuräumen. Dafür ist auch eine Sicherung des privaten Versicherungsmarktes notwendig, denn gerade er sichert wichtige Finanzierungsquellen - insbesondere für die Krankenhäuser - und so auch die Qualität der Leistungserbringung.

\section{Unternehmerische Freiräume schaffen}

Gesundheitspolitik ist auch immer Ordnungspolitik. Eine nachhaltige Ordnung im Gesundheitswesen wird dann gewährleistet, wenn erfolgreiche Prinzipien und Strategien der Vergangenheit mehr Raum erhalten und man mutig mit traditionellen Strukturen bricht, welche die Zukunftsfähigkeit gefährden. Der Kern des Erfolgs liegt in einem Mischsystem aus Leistung, Solidarität, Wettbewerb und sozialem Ausgleich. Letzteren kann es aber nur geben, wenn vorher erwirtschaftet wurde, was umverteilt werden soll. Dafür ist unternehmerischer Freiraum nötig. Privatwirtschaftliches Handeln ist ein Garant dafür.

„Vorbild sein für andere und zeigen, dass medizinische und pflegerische Höchstleistungen mit wirtschaftlicher Betriebsführung vereinbar sind." Dieser Unternehmensgrundsatz der Sana Kliniken könnte auch Leitmotiv für das gesamte Gesundheitswesen sein. Ohne die Anerkennung der Notwendigkeit wirtschaftlichen Denkens im Gesundheitswesen wird die Lösung der Probleme noch schwieriger.

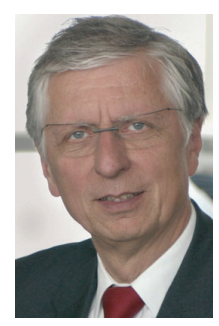

Korrespondenz

Dr. Reinhard Schwarz Sana Kliniken GmbH \& Co. KGaA Gustav-HeinemannRing 133 81739 München r.schwarz@sana.de 